\title{
Postscript on Viable Ground-States for Calculating Topological $\pi$-Electron Ring-Currents Using the Hückel-London-Pople-McWeeny Model
}

\author{
(D) Timothy K. Dickens, (1) Roger B. Mallion*
}

Peterhouse, Cambridge CB2 1RD, England, United Kingdom

* Corresponding author's e-mail address: mallionr29b@aol.com

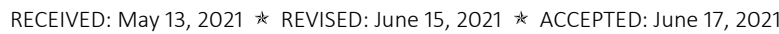

— This PaPer is Dedicated to Prof. Milan Randić on the ocCasion of his 90 ${ }^{\text {Th }}$ BirThday, AND to the Memory of Prof. Mircea Diudea —

\begin{abstract}
Attention is drawn to the idea that, in the context of the Hückel-London-Pople-McWeeny (HLPM) approach to $\pi$-electron ringcurrents, the basic Aufbau process can be mimicked by means of a graph-theoretical algorithm and that the outcome is determined solely by the order of the eigenvalues of the arbitrary molecular-graph representing an extant or hypothetical conjugated system. The Aufbau process usually results in a closed-shell ground-state, but sometimes a unique triplet ground-state arises, sometimes doublets, as well as unique groundstates of higher multiplicity, are encountered, and, on occasions, no uniquely defined $\pi$-electronic ground-state is established at all. Previously, the only examples of the latter ('pathological') case - which, as with triplet ground-states and other ground-states that are not singlets, precludes the possibility of any HLPM calculation - were graphs that are unlikely candidates for being extant or viable conjugated systems. In this note, however, an example is documented of what is, ostensibly, a plausible unsaturated structure - namely, (Coronene) ${ }^{6-}$. In the conclusion, attention is drawn to a procedure that averages electron occupation amongst the several orbitals of a degenerate shell.
\end{abstract}

Keywords: topological ring-currents in conjugated systems, $\pi$-electron triplet ground-states, Aufbau principle, ambiguous ground-states.

\section{INTRODUCTION}

$\mathbf{T}$ HIS contribution is concerned with the Hückel-LondonPople-McWeeny (HLPM) model of $\pi$-electron ringcurrents in conjugated systems. ${ }^{[1-3]}$ Professor Milan Randić - to whose $90^{\text {th }}$ birthday we are paying tribute via this article - has worked extensively, over a period of more than forty years, on a different model of ring currents, one that is based on his own approach ${ }^{[4]}$ of 'conjugated'[4] ('conjugation' ${ }^{[5,6]}$ ) circuits. $^{[7-10]}$

In the special publication that arose from MATH/CHEM/COMP 31 (Dubrovnik, 2019), we were privileged to honour the late $E$. C. Kirby by means of a paper ${ }^{[11]}$ entitled "Some Observations on Triplet Ground-States in the Context of 'Topological' (HLPM) Ring-Currents in Conjugated Systems." In that work, attention was drawn to the problems that arise in the HLPM approach when, on application of the Aufbau Principle, ${ }^{[12-17]}$ the $\pi$-electronic ground-state configuration of the hydrocarbon under study is found to be a triplet. ${ }^{[11]}$
Previous work ${ }^{[13,14,16]}$ had shown that the Aufbau process ${ }^{[12]}$ can be mimicked by means of a graph-theoretical[18] algorithm and that the outcome is determined solely by the order of the eigenvalues of the molecular-graph ${ }^{[18]}$ representing the conjugated system under study. It was further emphasised that application of the Aufbau Principle ${ }^{[12]}$ to the $\pi$-electron energy-levels corresponding to the eigenvalues of a graph - taken to represent the carbon-atom connectivity of an extant or hypothetical conjugated hydrocarbon - leads usually to the establishment of a unique closed-shell ground-state - that is, a singlet; in such circumstances a ring-current calculation by means of the HLPM approach ${ }^{[1-3]}$ may be carried out satisfactorily. ${ }^{[11]}$ This is the case in, for example, Figure 2 of Ref. [13].

Sometimes, however, a triplet ground-state arises this presents the difficulty in calculating HLPM ringcurrents that is addressed in Ref. [11]. Such a situation is encountered, for example, in the simple treatment of the $[4 n]$-annulenes without bond alternation, in which infinite 
paramagnetism would, in principle, be predicted by the HLPM approach. ${ }^{[19,20]}$ A more realistic example is the extant hydrocarbon of the [8]-circulene family shown as structure 4 on the right-hand side of Figure 2 in Ref. [11] - a structure that was recently considered by Baryshikov et al.[21]

Ground states of even higher multiplicities can also occasionally be found in the case of more bizarre molecular graphs that are, however, unlikely to represent viable molecules - such as, for example, that depicted in Figure 2 of Ref. [13]. Another, somewhat chemically more realistic, example was, however, recently encountered in the case of the neutral 'altan'[3] shown in Figure 1. When the Aufbau process ${ }^{[12]}$ is applied to the neutral species of this structure the uniquely defined $\pi$-electron ground-state involves no fewer than four singly occupied non-bonding orbitals displaying electrons with four parallel spins (thereby constituting what might be called a quintuplet groundstate, because the magnitude $(|s|)$ of the total spin $(s)$ is $(1 / 2+1 / 2+1 / 2+1 / 2)=2$, so that the multiplicity, $N-$ from the expression ${ }^{[22]} N=2|s|+1-$ is $\left.(2 \times 2)+1=5\right)$.

Finally, it was observed by one of the present authors many years ago ${ }^{[13-16]}$ that, sometimes, no uniquely defined $\pi$-electronic ground-state is obtainable at all when the Aufbau process ${ }^{[12]}$ is applied. To achieve an example of the latter case, however, a somewhat outré 'molecular' graph had to be devised - explicitly and variously depicted in Figure 3 of Ref. [13], in Figure 8 of Ref. [14], in structure (I) in Ref. [15] and in Figure 8 of Ref. [16]. The graph in question is the Octahedral Graph - which may be visualised in three dimensions as a bipyramid (octahedron) ${ }^{[23]}-$ depicted here

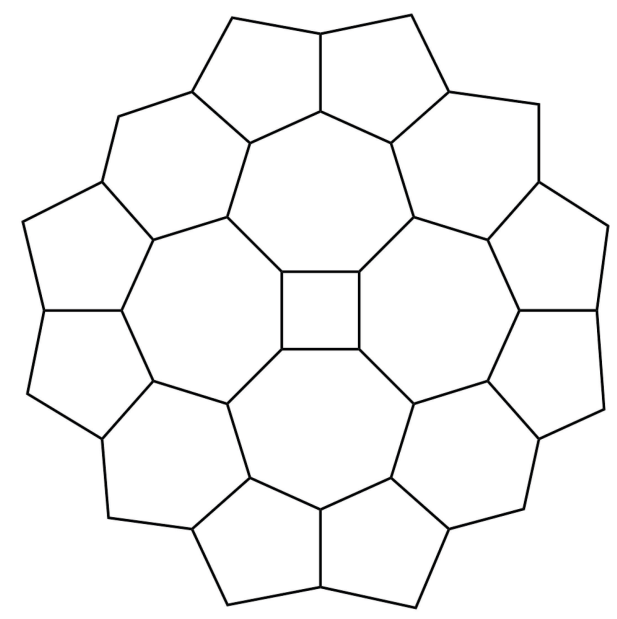

Figure 1. The molecular graph[18] of a neutral 'altan'[3] structure which, when the Aufbau process ${ }^{[13,14,16]}$ is applied to it, gives rise to a unique $\pi$-electron ground-state configuration in which four quadrupally degenerate HOMO non-bonding orbitals are each singly occupied - thereby presenting a total of four electrons with parallel spins and formally leading to a quintuplet ground-state.[22]

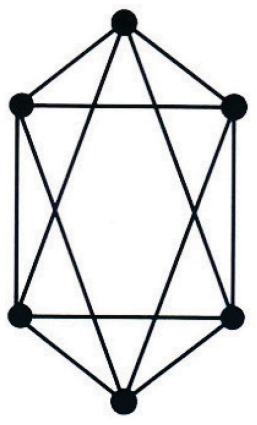

Figure 2. The Octahedral Graph ${ }^{[23]}$ which, when considered as a 'molecular graph',[18] gives rise to an ill-defined $\pi$-electron ground-state when the Aufbau process ${ }^{[13,14,16]}$ is applied to it. This has been referred to as an example of the 'ambiguous case' in Refs. [13-15], and as the 'pathological case' in Ref. [16].

in Figure 2. Application of the Aufbau process to the Octahedral Graph, when it is (unrealistically) regarded as a molecular graph, ${ }^{[18]}$ is considered in the next section.

\section{THE FAILURE OF THE AUFBAU PROCESS WHEN APPLIED TO THE OCTAHEDRAL GRAPH}

The eigenvalue list ${ }^{[23]}$ for the Octahedral Graph ${ }^{[23]}$ (Figure 2) - which consists entirely of integers - is $4,0,0,0,-2,-2$. In the Hückel molecular orbital interpretation ${ }^{[24]}$ this means that there are considered to be six 'energy levels' which are, in non-decreasing order of energy: $(\alpha+4 \beta), \alpha, \alpha, \alpha$, $(\alpha-2 \beta),(\alpha-2 \beta)$, where $\alpha$ and $\beta$ are the standard Hückel parameters. ${ }^{[24]}$ These are shown pictorially in Figure 3 of Ref. [13], in Figure 8 of Ref. [14] and in Figure 8 of Ref. [16]. The coefficients of $\beta$ are explicitly listed - numbered 1-6 in increasing order of energy (as, it will be recalled, $\beta$ is negative $\left.{ }^{[24]}\right)$ - in Scheme 1.

With six $\pi$-electrons to distribute amongst these six 'energy levels', the first two would be assigned, with paired spins, to the unique Level 1 , of energy $(\alpha+4 \beta)$, thereby completely filling this bonding orbital. On proceeding up the list, the next encountered are the three degenerate non-bonding orbitals labelled 2, 3, and 4. Completely to fill these would require six more $\pi$-electrons but in fact only four of the original six remain, and are still available to be assigned. As Scheme 1 shows, there is no unambiguous way to do this; there are in fact ${ }^{3} \mathrm{C}_{1}$ ways to complete the Aufbau process - namely, according to the Aufbau rules: ${ }^{[12]}$ (a) Level 2 doubly occupied and Levels 3 and 4 singly occupied (as in column (a) of Scheme 1); (b) Level 3 doubly occupied and Levels 2 and 4 singly occupied (as in the middle column of Scheme 1); (c) Level 4 doubly occupied and Levels 2 and 3 singly occupied (as in column (c) of Scheme 1). 


$\begin{array}{cccccc}6 & -2 & 6 & -2 & 6 & -2 \\ 5 & -2 & 5 & -2 & 5 & -2 \\ 4 & 0 & 4 & 0 & 4 & 0 \\ 3 & 0 & 3 & 0 & 3 & 0 \\ 2 & 0 & 2 & 0 & 2 & 0 \\ 1 & 4 & 1 & 4 & 1 & 4\end{array}$

(a)

(b)

(c)

Scheme 1. Three possible endings ((a), (b), and (c)) to the Aufbau process (involving the Pauli Exclusion Principle and Hund's Rules of Maximum Multiplicity ${ }^{[12]}$ when it is schematically applied to the Octahedral Graph (Figure 2). The left-hand column of each of the three sections (a), (b), and (c) labels the energy levels (in increasing order, numbered 1-6) and the right-hand column in each section documents the value of $k$ in the expression of a Hückel energy-level as $(\alpha+k \beta)$. (Thus, for example, energy-level number 5 has energy $(\alpha-2 \beta)$.) In the right-hand column of (a), (b) and (c), red indicates doubly occupied orbitals, green singly occupied, and black unoccupied energy-levels.

Such a situation has been designated as the ambiguous case' in Refs. [13] and [14], and as 'the pathological case' in Ref. [16].

Of course, this Octahedral Graph (Figure 2) is very unlikely to be the molecular graph ${ }^{[18]}$ of any extant or possibly viable molecule. However, in the course of our HLPM studies of Corannulene and Coronene and several of their anions, we serendipitously came across the first example that we had encountered of a structure that, on the face of it, could potentially exist, but in fact turned out to be an example of this 'ambiguous case'[13-15] ('pathological case $^{\prime[16]}$ ) in which a unique ground-state configuration could not be established on application of the Aufbauprinzip. ${ }^{[12]}$ This structure is the hexa-anion of Coronene - (Coronene) $)^{6-}$ - to which we now turn.

\section{THE FAILURE OF THE AUFBAU PROCESS WHEN APPLIED TO (CORONENE) $^{6-}$}

Although at various times we had both previously studied neutral Corannulene ${ }^{[25,26]}$ and Coronene, ${ }^{[26]}$ we were motivated by Monaco and Zanasi's definitions ${ }^{[27,28]}$ of the altans of these structures (subsequently extended and mathematically generalised by Gutman ${ }^{[2,30]}$ and by Bašić et al. ${ }^{[31,32]}$ to treat these novel conjugated systems by the HLPM approach ${ }^{[1-3]}$ - the neutral altans, ${ }^{[33]}$ as well as their respective di-anions ${ }^{[34]}$ and hexa-anions. ${ }^{[35]}$ This led us to study the 'parents' of these altans in their own right - that is, Corannulene and Coronene themselves, together with their various oxidation states. We now demonstrate that
(Coronene) $^{6-}$ is a 'pathological case' in that it is seen to be an example of an ambiguously defined $\pi$-electronic groundstate when the Aufbauprinzip is invoked. ${ }^{[12]}$ The energy levels (labelled 1-24) for the Coronene molecular-graph are shown in Scheme 2 (which has the same colour conventions about occupied (red) and unoccupied (black) energy-levels as Scheme 1, and employs blue to denote those orbitals whose occupancy will - in the course of applying the Aufbau process[ ${ }^{[12]}$ - be seen to be ambiguous).

Neutral Coronene has $24 \pi$-electrons and so, in the hexa-anion, there are $30 \pi$-electrons to be distributed in the energy levels listed in Scheme 2, by invoking the Aufbau process. ${ }^{[12]}$ The first 28 of these will doubly fill each of Levels 1-14 (coloured in red, in Scheme 2). Then there are two $\pi$-electrons left but they have to be distributed into the next-highest level - which, however, is triply degenerate (that is, there are three repeated eigenvalues), with the next three levels $(15,16$ and 17) all being of energy 1.0000000 (coloured in blue, in Scheme 2). By analogy, therefore, with the case of the Octahedral Graph just considered in Scheme 1, there are three $\left({ }^{3} \mathrm{C}_{2}\right)$ possible ways of assigning the last two electrons to the three degenerate

$\begin{array}{rr}24 & -2.6751309 \\ 23 & -2.2143197 \\ 22 & -2.2143197 \\ 21 & -1.6751309 \\ 20 & -1.6751309 \\ 19 & -1.5391889 \\ 18 & -1.2143197 \\ 17 & -1.0000000 \\ 16 & -1.0000000 \\ 15 & -1.0000000 \\ 14 & -0.5391889 \\ 13 & -0.5391889 \\ 12 & 0.5391889 \\ 11 & 0.5391889 \\ 10 & 1.0000000 \\ 9 & 1.0000000 \\ 8 & 1.0000000 \\ 7 & 1.2143197 \\ 6 & 1.5391889 \\ 5 & 1.6751309 \\ 4 & 1.6751309 \\ 3 & 2.2143197 \\ 2 & 2.2143197 \\ 1 & 2.6751309\end{array}$

Scheme 2. The Aufbau process schematically applied to (Coronene) ${ }^{6-}$. Red indicates doubly occupied orbitals, black denotes unoccupied energy-levels and blue emphasises those energy levels whose occupancy will be seen to be ambiguous. 


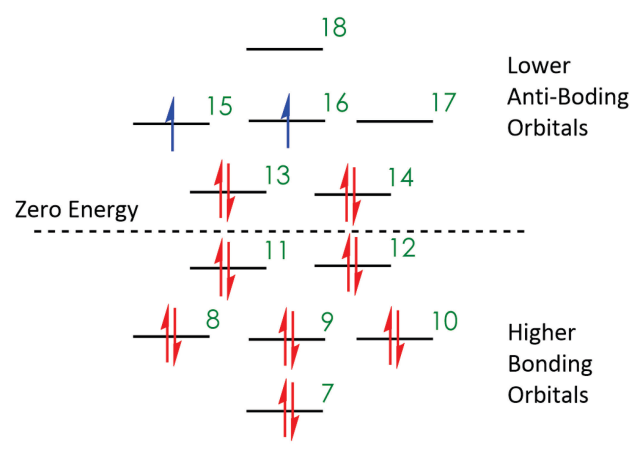

Figure 3. Schematic diagram depicting the frontier orbitals numbered according to Scheme 2 - of (Coronene) ${ }^{6-}$, together with their partial occupancy, as described in the text.

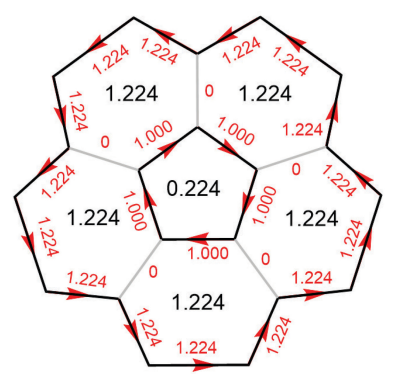

Neutral Corannulene energy-levels 15-17 - (a) Levels 15 and 16 singly occupied and Level 17 unoccupied (as in the schematic diagram of the frontier orbitals of (Coronene ${ }^{6-}$ ) presented in Figure 3); (b) Levels 15 and 17 singly occupied and Level 16 unoccupied; (c) Levels 16 and 17 singly occupied and Level 15 unoccupied. Hence, the 'pathological case' is again encountered but, this time, it is associated with the molecular graph ${ }^{[18]}$ of what would appear, on the face of it, to be a viable unsaturated structure, rather than just a somewhat fanciful abstract graph such as the illustrative example in Refs $[13,14,16]$ - which is unlikely to be the molecular graph of any realistically potential conjugated system. We thus observe that the 'pathological' problem arises with (Coronene) ${ }^{6-}$ but (as will be seen in the next section) not with the analogous (Corannulene) ${ }^{6-}$.

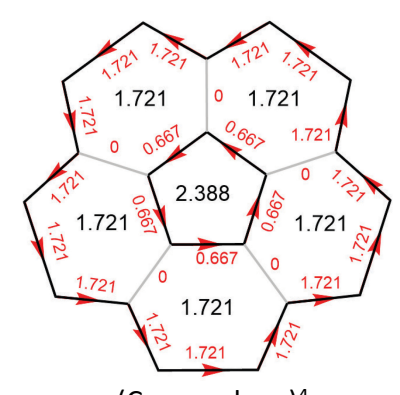

(Corannulene) $^{4-}$

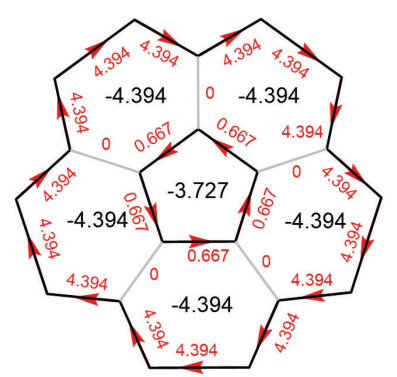

(Corannulene) $^{6-}$

Figure 4. HLPM Ring-current and bond-current maps for neutral Corannulene ${ }^{[25,26]}$ and its tetra- and hexa-anions. Figures in black in the centres of rings denote the corresponding ring-current intensities (expressed as a ratio to the benzene value); positive ring-currents are diamagnetic and are considered to flow in the anti-clockwise direction around their respective rings, whilst negative ring-currents are paramagnetic and flow in the clockwise direction. Red figures written along the bonds denote the bond-currents (in the directions indicated by the arrows); these bond currents are compliant with Kirchhoff's Law of currents at junctions ${ }^{[10,36]}$ and are, thereby, consistent with these ring currents.

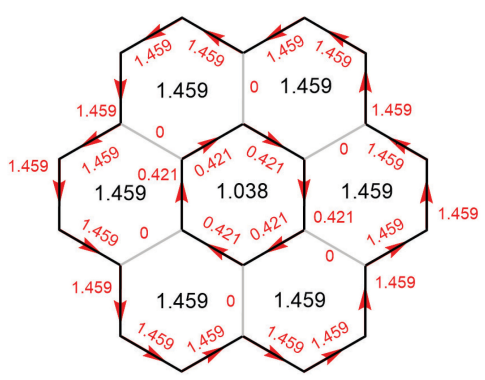

Neutral Coronene

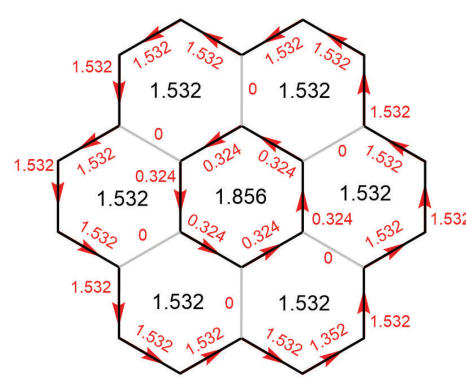

(Coronene) $)^{4-}$

Figure 5. HLPM Ring-current and bond-current maps for neutral Coronene ${ }^{[26]}$ and its tetra-anion. For the conventions on displaying ring currents and bond currents please see the caption to Figure 4. 
Table 1. Direction of Current Flow Around the Central Ring and the Perimeter of Neutral and Anionic Corannulenes and Coronenes.

\begin{tabular}{|c|c|c|}
\hline Structure & $\begin{array}{l}\text { Nature of Current Flow } \\
\text { Around the Central Ring }\end{array}$ & $\begin{array}{l}\text { Nature of Current Flow } \\
\text { Around the Perimeter }\end{array}$ \\
\hline Corannulene (Neutral) & Paratropic & Diatropic \\
\hline (Corannulene) $)^{4-}$ & Diatropic & Diatropic \\
\hline$(\text { Corannulene })^{6-}$ & Diatropic & Paratropic \\
\hline Coronene (Neutral) & Paratropic & Diatropic \\
\hline (Coronene) $^{4-}$ & Diatropic & Diatropic \\
\hline
\end{tabular}

\section{HLPM RING-CURRENTS AND BOND-CURRENTS IN CORANNULENE AND CORONENE AND SOME OF THEIR MULTI-ANIONS}

Because we had previously studied the altans of Corannulene and Coronene ${ }^{[33]}$ and several of their multianions, ${ }^{[34,35]}$ we resolved to examine the HLPM properties of the 'parents' ${ }^{[27]}$ and so wished to perform calculations on the neutral species, the di-anions, the tetra-anions, and the hexaanions of both Corannulene and Coronene. In practice, however, of the eight potential species - neutral Corannulene,

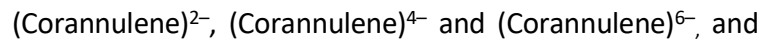
neutral Coronene, (Coronene) $)^{2-}, \quad$ (Coronene) $)^{4-}$ and (Coronene) $^{6-}-$ only five of them did not give problems with $\pi$-electronic ground-states when the Aufbau process ${ }^{[12]}$ was applied. The dianions of both species exhibited the triplet ground-state' problem, ${ }^{[11]}$ whilst, as was seen in the previous section, (Coronene) ${ }^{6-}$ gave rise to the 'pathological' case, because there is no unique $\pi$-electronic ground-state of any kind when the Aufbau process is invoked.

Figure 4 displays the HLPM ring currents and bond currents of neutral Corannulene, (Corannulene) ${ }^{4-}$, and

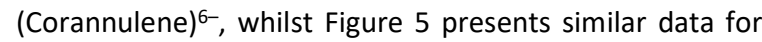
neutral Coronene and (Coronene) $)^{4-}$. The methods of calculation and the tenants of the HLPM model - including matters concerning planarity of the carbon-atom network - are all exactly as in the section labelled 'The overall structure of HLPM ring-current calculations' in Ref. [11] and as described under the heading 'Calculations' in Ref. [35]; they are, accordingly, not repeated here.

Table 1 summarises what Figures 4 and 5 show regarding the magnetic nature (diatropic/paratropic) of the current flows (a) around the central ring, and (b) around the perimeter, of the five structures on which we were able to perform the calculations illustrated in Figures 4 and 5 .

It may be observed that not all four of the permutations of 'Paratropic/Diatropic' appear here - only three of them do. 'Paratropic/Diatropic' occurs twice and 'Diatropic/Diatropic' also occurs twice. 'Diatropic/Paratropic' occurs once, but the missing permutation ('Paratropic/Paratropic') does not arise in those five species on which calculation has been possible.
Before stating our concluding remarks, we just enter a small caveat by addressing an astute question raised by a Referee: answering it may prevent other readers from being similarly disconcerted. It was asked: 'Why are the figures in the central rings of Figures 4 and 5 for neutral Corannulene and Coronene, respectively, positive? In these rings the current is clockwise then, according to the captions, they should be negative.'

The answer to this question is materially connected with the fact that the 'ring current' in a microscopic conjugated system is the equivalent of the 'loop current' ${ }^{[36-39]}$ that flows around an enclosed area in a macroscopic electrical network of the classic type to which Kirchhoff's Laws apply. ${ }^{[36-39]}$ The 'bond currents' in the microscopic conjugated system correspond to the currents in the individual wires that comprise the traditional macroscopic electrical network. ${ }^{[36-39]}$ Furthermore, in a general conjugated system, the magnitude and direction of the bond current in a bond that is shared between two rings is the algebraic resultant of a competition between the ring currents in the rings that lie either side of that bond.

Consider, for example, any one of the peripheral rings in Coronene - say, the left-hand ring of the pair at the top of the structure when Coronene is oriented as depicted in the left-hand side of Figure 5. This ring is characterised by a ring-current intensity of 1.459 , in the anti-clockwise sense around that peripheral ring. For any bond in this ring that is unshared with any other ring, the bond current would, accordingly, also be 1.459, in the direction shown in Figure 5. This is the case with the three bonds in each peripheral ring that lie along the perimeter of Coronene. Now, in addition, one bond in each of these peripheral rings is shared with the central ring, which itself is characterised by a diamagnetic (anti-clockwise) ringcurrent of intensity 1.038; if this central ring were completely isolated, sharing no bonds with any other rings, then 1.038 would also be the bond-current flowing anticlockwise along each of the bonds of the central ring. However, as seen from Figure 5, each bond in the central ring (which bears a ring-current of intensity 1.038) is in fact shared with a peripheral ring bearing a ring-current of 1.459. The smaller diamagnetic ring-current (1.038) which would otherwise flow anti-clockwise around the central 
ring is, thereby, overwhelmed by the larger current of 1.459 flowing in the anti-clockwise sense around each peripheral ring. The net current in each of the shared bonds in the central ring is thus of magnitude $1.459-1.038=0.421$, in a direction which is anti-clockwise around the peripheral ring and clockwise around the central ring.

Hence, we are able to reconcile the ostensible paradox that - understandably - troubled the Referee: namely, that the net circulation around the bonds of the central ring in Coronene is observed, by the above arguments, to be in the clockwise (paramagnetic) sense (as indicated by the arrows in the left-hand part of Figure 5), despite the fact that the central ring is itself formally characterised, overall, by a (positive) diamagnetic ringcurrent (as is also shown in Figure 5.)

An analogous argument may be invoked in order to explain the clockwise net-flow and the diamagnetic ring-current encountered in the central ring of neutral Corannulene (as shown in the first ring-current/bondcurrent map presented in Figure 4).

\section{FURTHER DISCUSSION}

The calculations reported in Figures 4 and 5 are merely the harbinger of a much more wide-ranging study that we are currently undertaking in which the HLPM approach ${ }^{[1-3]}$ is applied to a variety of extant and hypothetical conjugated systems. One of the aims of this extended project is to establish what factors determine the direction of flow around various cycles of the structures examined; the conclusions from this will be reported in detail in a later publication.

The object of the present note has been more modest - namely, to add a postscript to the comments that we made in Ref. [11] about how what we have called 'the triplet ground-state problem' foils HLPM calculations when it arises. We have drawn attention here to the idea that the basic Aufbau process ${ }^{[11]}$ can be mimicked by means a graphtheoretical algorithm ${ }^{[13,14,16]}$ and that the outcome is determined solely by the order of the eigenvalues of the arbitrary molecular-graph under study. This usually results in a closed-shell ground-state, but sometimes triplet groundstates arise, and sometimes doublets, in addition to welldefined ground-states of even higher multiplicity, present themselves - and, only comparatively rarely, there are even cases in which there is no uniquely defined $\pi$-electronic ground-state at all; these latter eventualities we refer to as 'ambiguous' or 'pathological' cases. ${ }^{[13,14,16]}$ Previously, the only examples of such pathological cases that had emerged were graphs that are unlikely candidates for being extant or potentially viable conjugated systems. ${ }^{[13,14,16]}$ In this note, however, we have documented an example of a pathological case that, ostensibly, is a plausible candidate for being a viable unsaturated structure - namely, (Coronene) ${ }^{6-}$.

\section{CONCLUSION}

In conclusion, we point out that the difficulties that are encountered as a result of the present discussion, and of that of Ref. [11], arise because the McWeeny formalism ${ }^{[0,41]}$ - the equations of which are directly incorporated into the HLPM approach $^{[1-3]}$ - explicitly assumes that closed-shell systems are being dealt with. ${ }^{[40,41]}$ We point out, however, that Fowler et al. ${ }^{[39]}$ have recently documented a scheme in which a calculation based on the Hücke[ ${ }^{[24]}$-London ${ }^{[43]}$ method may be carried out in what they call[42] a 'fractional-occupation approximation'. In this process, each orbital in a shell of $p$ orbitals that has a total occupation of $q$ electrons is assigned an occupation number, $q / p$, which is possibly fractional; on this model, the resulting electronic occupation in the shell in question could, in some sense, be thought of as being, thereby, 'smeared' or 'averaged' over all the orbitals ( $p$ in number) in that shell. ${ }^{[42]}$ This invites future investigation.

Acknowledgment. We should like to pay tribute, on the occasion of his $90^{\text {th }}$ birthday, to one of the honorands of MATH/CHEM/COMP 32, Professor Milan Randić, and to thank him for his friendly correspondence on ring currents, and his unfailingly convivial company at conferences, over the years. We are also grateful to an anonymous Referee whose careful reading of the manuscript caused us to consider an apparent contradiction - which we were able successfully to resolve in the above final text. Furthermore, RBM thanks the Master and Fellows of Peterhouse, Cambridge for his election as a Visiting Fellow, which has facilitated collaboration with TKD.

\section{REFERENCES}

[1] R. B. Mallion, Croat. Chem. Acta 2008, 81, 227-246.

[2] A. T. Balaban, T. K. Dickens, I. Gutman, R. B. Mallion, Croat. Chem. Acta 2010, 83, 209-215.

[3] T. K. Dickens, R. B. Mallion, MATCH Commun. Math. Comput. Chem. 2016, 76, 297-356.

[4] M. Randić, Chem. Phys. Letters 1976, 38, 68-70. https://doi.org/10.1016/0009-2614(76)80257-6

[5] J. A. N. F. Gomes, Some Magnetic Properties of Molecules, D. Phil. Thesis, University of Oxford, 1976, especially pp 69-73.

[6] J. A. N. F. Gomes, R. B. Mallion, Revista Portuguesa de Química 1979, 21, 82-89.

[7] J. Gayoso, A. Boucekkine, Comptes. Rend. Acad. Sci. (Paris) 1979, C205, 327-330.

[8] M. Randić, Chem. Rev. 2003, 103, 3449-3605, especially pp 3539-3543. https://doi.org/10.1021/cr9903656

[9] M. Randić, Chem. Phys. Letters 2010, 500, 123-127. https://doi.org/10.1016/j.cplett.2010.09.064

[10] T. K. Dickens, J. A. N. F. Gomes, R. B. Mallion, J. Chem. Theory Computation 2011, 7, 3661-3674, especially §3, pp 3665-3668. https://doi.org/10.1021/ct2002539 
[11] T. K. Dickens, R. B. Mallion, Croat. Chem. Acta 2019, 92 445-455. https://doi.org/10.5562/cca3538

[12] F. A. Cotton, Chemical Applications of Group Theory; John Wiley \& Sons: New York \& London, 1963, pp 123-125.

[13] R. B. Mallion, D. H. Rouvray, Mol. Phys. 1978, 36 125-128. https://doi.org/10.1080/00268977800101451

[14] R. B. Mallion, D. H. Rouvray, Studia Scientiarum Mathematicarum Hungarica 1978, 13, 229-243.

[15] R. B. Mallion, Croat. Chem. Acta 1983, 56, 477-490.

[16] R. B. Mallion, 'The topological nature of the Aufbau process and of quantities calculated via simple molecular-orbital theory', in: A. Graovac, I. Gutman, D. Vukičević (Eds.), Mathematical Methods and Modelling for Students of Chemistry and Biology; Hum naklada d.o.o.: Zagreb, 2009, pp 55-86, especially $\S 6$ and $\S 7$, pp 72-81.

[17] A. Ceulemans, E. Lijnen, P. W. Fowler, R. B. Mallion, T. Pisanski, Proc. Royal Soc. (London), Series A 2012, 468, 971-989.

https://doi.org/10.1098/rspa.2011.0508

[18] N. Trinajstić, Chemical Graph Theory, $2^{\text {nd }}$ ed.; CRC Press: Boca Raton, Florida, USA, 1992.

[19] J. A. Pople, K. G. Untch, J. Am. Chem. Soc. 1966, 88, 4811-4815. https://doi.org/10.1021/ja00973a009

[20] J. Aihara, Phys. Chem. Chem. Phys. 2016, 18, 1184711857, especially p. 11853. https://doi.org/10.1039/C5CP06471F

[21] G. B. Baryshikov, R. R. Valiev, N. N. Karaush, D. Sundholm, B. F. Mineav, Phys. Chem. Chem. Phys. 2016, 18, 8980-8992.

https://doi.org/10.1039/C6CP00365F

[22] I. N. Levine, Quantum Chemistry, $4^{\text {th }}$ ed.: Englewood Cliffs, New Jersey, USA \& Prentice Hall College Division: Upper Saddle River, New Jersey, USA, 1991.

[23] D. M. Cvetković, M. Doob, H. Sachs, Spectra of Graphs: Theory and Applications, $3^{\text {rd }}$ ed.; Johann Ambrosius Barth Verlag: Heidelberg \& Leipzig, 1995, especially Table 4, Graph 4.37, pp 310 \& 311.

[24] C. A. Coulson, B. O'Leary, R. B. Mallion, Hückel Theory for Organic Chemists; Academic Press: London, 1978, especially Appendix A, pp 148-150.

[25] R. B. Mallion, 'Ring currents in Corannulene, a prototype pattern-molecule for Buckminsterfullerene', in: Math/Chem/Comp 1988. A. Graovac (Ed.). Proceedings of an International Course and Conference on the Interfaces between Mathematics and Computer Science, Dubrovnik, Yugoslavia, $20^{\text {th }}$ $25^{\text {th }}$ June, 1988, Studies in Physical and Theoretical Chemistry, vol. 63, Elsevier Science Publishers: B.V., Amsterdam, 1989, pp 505-510.
[26] T. K. Dickens, R. B. Mallion, Chem. Phys. Letters 2011 517, 98-102.

https://doi.org/10.1016/j.cplett.2011.10.002

[27] G. Monaco, R. Zanasi, J. Phys. Chem. A 2012, 116, 9020-9026. https://doi.org/10.1021/jp302635j

[28] G. Monaco, R. Zanasi, J. Phys. Org. Chem. 2013, 26 , 730-736. https://doi.org/10.1002/poc.3117

[29] I. Gutman, J. Serb. Chem. Soc. 2014, 79, 1515-1521. https://doi.org/10.2298/JSC140619080G

[30] I. Gutman, Iran. J. Math. Chem. 2014, 5, 85-90. https://doi.org/10.30875/f9d299c3-en

[31] N. Bašić, T. Pisanski, MATCH Commun. Math. Comput. Chem. 2015, 74, 645-658.

[32] N. Bašić, P. W. Fowler, T. Pisanski, J. Math. Chem. 2016, 54, 977-1009.

https://doi.org/10.1007/s10910-016-0599-6

[33] T. K. Dickens, R. B. Mallion, J. Phys. Chem. A 2014, 118, 933-939. https://doi.org/10.1021/jp411524k

[34] T. K. Dickens, R. B. Mallion, J. Phys. Chem. A 2018, 122, 7666-7678.

https://doi.org/10.1021/acs.jpca.8b06862

[35] T. K. Dickens, R. B. Mallion, J. Phys. Chem. A 2020, 124, 7973-7990.

https://doi.org/10.1021/acs.jpca.0c04606

[36] E. C. Kirby, R. B. Mallion, P. Pollak, P. J. Skrzyński, Croat. Chem. Acta 2016, 89, 403-417. https://doi.org/10.5562/cca2995

[37] J. A. N. F. Gomes, R. B. Mallion, 'The concept of ring currents', in: Concepts in Chemistry. D. H. Rouvray (Ed.), Research Studies Press Limited: Taunton, Somerset, England, United Kingdom, 1997, and John Wiley \& Sons. Inc.: New York, 1997, Chapter 7, pp 205-253.

[38] H. M. S. Cundy, S. M. P. Advanced Mathematics Book 3 (Metric), Cambridge University Press: London, 1970, pp 911-912.

[39] D. Smart, Linear Algebra \& Geometry, S. M. P. Further Mathematics Series, Cambridge University Press: Cambridge, England, United Kingdom, 1988. pp 307-308.

[40] R. McWeeny, Mol. Phys., 1958, 1, 311-321. https://doi.org/10.1080/00268975800100381

[41] R. B. Mallion, Proc. Royal Soc. (London) Series A 1974/1975, 341, 429-449, especially the Appendix, pp 446-447.

https://doi.org/10.1098/rspa.1975.0002

[42] P. W. Fowler, W. Myrvold, C. Gibson, J. Clarke, W. H. Bird, J. Phys. Chem. A 2020, 124, 4717-4533, especially p. 4528. https://doi.org/10.1021/acs.jpca.0c02748

[43] F. London, J. Phys. Radium (7ème Série) 1937, 8, 397-409.

https://doi.org/10.1051/jphysrad:01937008010039700 